\title{
DYNAMIC LAYOUT OF VISUAL SUMMARIES FOR SCALABLE VIDEO
}

\author{
Janko Ćalić, Marta Mrak and Ahmet Kondoz \\ I-Lab, Centre for Communication System Research \\ University of Surrey, Guildford, United Kingdom \\ \{j.calic, m.mrak, a.kondoz\}@surrey.ac.uk
}

\begin{abstract}
The paper brings a novel method for generating visual summaries of scalable videos. The generated summaries can dynamically adapt to requirements defined by display size, user's needs or channel limitations. It utilises compressed domain features coupled with efficient contour evolution algorithm in order to generate a scale space of temporal video descriptors. The layout of the visual summary is created using an efficient graph clustering technique and a fast discrete optimisation algorithm, enabling dynamic video summarisation in real-time. The experimental results show good scalability of the dynamic layout and highly efficient generation of visual summaries.
\end{abstract}

Index Terms - discrete optimisation, video analysis, video summarisation, scalable video coding

\section{INTRODUCTION}

Being one of the central requirements of Future Internet Multimedia, real-time interactivity and responsiveness of the multimedia interfaces is a prime goal in multimedia management research. Nowadays, user experience is limited to a rather unidirectional delivery of temporal media, delivered over the Internet or stored locally. This problem emerges especially in the case of large data repositories. In order to facilitate intuitive and responsive interaction with large multimedia collections, the issues of system efficiency and usability need to be addressed.

The work presented in this paper introduces an efficient system for large-scale video summarisation that exploits compressed-domain analysis of scalable video. Aimed at responsive and intuitive browsing interfaces for large video databases, the system generates visual representation of video data in a form of a comic-like summary with low latency, thus making a shift towards more user-centred summarisation and browsing of large video collections by augmenting user's interaction with the content rather than learning the way users create related semantics.

The work presented was developed within VISNET II, a European Network of Excellence, funded under the European Commission IST FP6 programme.
In generating the visual summaries, presented algorithm follows the narrative structure of comics, linking the temporal flow of video sequence with the spatial position of panels in a comic strip. This approach differentiates our work from the typical reverse storyboarding [1] or video summarisation approaches. Although there have been attempts to utilise the form of comics as a medium for visual summarisation of videos [2] [3], the high complexity of these algorithms hindered summarisation at the larger scale or with low processing latency. In order to overcome the demanding complexity constraints, the proposed system utilises video analysis algorithm that uses compressed-domain hierarchical motion information, coupled with a fast discrete optimisation algorithm for creation of the comic-like layout of extracted key-frames. Avoiding full video decoding, the analysis algorithm decompresses only the motion information from targeted temporal decomposition level of the scalable video. The motion activity metric is chosen for capturing intensity of action since it is highly correlated to human perception [4]. Using a fast and robust geometrical curve simplification algorithm, a set of most representative key-frames is extracted as a visual summary of the analysed video. In order to generate an intuitive and yet compact video browsing interface, our approach introduces a novel solution based on dynamic programming (DP). In addition, the presented algorithm applies a new approach to the estimation of key-frame sizes in the final layout by exploiting an efficient graph clustering methodology coupled with a specific cost function that balances between good content representability and discovery of unanticipated content. The evaluation results compared to existing methods of video summarization showed substantial improvements in terms of algorithm efficiency.

The utilisation of scalable video coding technology is presented in Section 2 followed by the description of an algorithm for fast selection of the most representative keyframes. Section 3 introduces a novel method for forming of video summaries that optimises generated visual representation to available spatial resources. The results of the algorithms presented are given in Section 4, followed by the final conclusions. 


\section{COMPRESSED DOMAIN KEY-FRAME EXTRACTION}

The proposed video analysis is performed utilising a motion activity measure. The motion activity descriptor is a standard tool for capturing intensity of action correlated to human perception [4]. In our work, the extraction of motion activity descriptor is performed from the layered bit-streams in scalable video.

The algorithmic complexity of the activity measure computation is very low due to its direct extraction form the compressed domain data. The motion information available from the compressed video is obtained using only partial decoding. The final selection of key-frames is based on evaluation of the motion activity metric, comparing the values of the metric for neighbouring frames and the overall video sequence activity.

\subsection{Compressed domain video analysis}

The activity metric for each frame is extracted from associated motion information generated in the process of video coding. Decoding of motion information without complete video decoding is a low-complexity process, which can be further simplified, if the layered video, such as scalable coded video [5] [6] [7], is used. This is due to the underlying hierarchical layered structure of the compressed stream. For bit-streams that are encoded using motion compensated temporal filtering [5] the motion information can be obtained for each compensated frame from different levels of temporal filtering. Lower temporal bit-stream layers consist of the data related to more distant frames. While in the context of compression the coding efficiency drops when the frames are more distant, those frames are still close enough for analysis. Therefore the analysis for key-frame selection can be performed from lower bit-stream layers.

In order to obtain the motion activity metric for the observed frame, a variance of magnitude of motion vectors is calculated for all temporal prediction modes. For bidirectional prediction the motion vector magnitudes are averaged and treated as the magnitudes for unidirectional prediction. The overall activity measure, $\alpha_{t}$ for a frame at time position $t$ is computed for all motion compensated frames at the lowest accessible bit-stream layer which is then used in keyframe selection algorithm described in the following section.

\subsection{Discrete contour evolution and key-frame extraction}

In order to generate a scalable temporal descriptor that facilitates dynamic extraction of key-frames from the sequence, the activity metric needs to be simplified in a way that spurious and small changes are discarded without any influence to the main features of the metrics curve. A method called Discrete Curve Evolution (DCE) [8] efficiently achieves this requirement: it leads to the simplification of curve complexity with no peak rounding effects and no dislocation of relevant features. The curve evolution process is guided by a relevance measure $K$, which is stable with respect to noisy deformations, and is given as:

$$
K_{i}=\left|\left(a_{i}-a_{i-1}\right) \cdot\left(t_{i}-t_{i-1}\right)\right|+\left|\left(a_{i+1}-a_{i}\right) \cdot\left(t_{i+1}-t_{i}\right)\right|,
$$

where $a_{i}$ are motion activity values at time indices $t_{i}$ at the particular DCE simplification stage. Initial values of $a_{i}$ correspond to $\alpha_{i}$, i.e. to the motion activity metrics for each analysed frame. The relevance measure $K_{i}$ is proportional to a change of area below the motion activity curve caused by the removal of the point $i$ on the curve. At each stage of simplification the relevance measure $K_{i}$ is iteratively updated. The optimal complexity level of the temporal descriptor is calculated as:

$$
\mathrm{K}_{\mathrm{opt}}=\overline{\mathrm{K}}-\log (\kappa) \cdot \sigma_{\mathrm{K}}
$$

where $\overline{\mathrm{K}}$ is the mean of all $K$ at different simplification stages, $\sigma_{\mathrm{K}}$ is its standard deviation, while the parameter $\kappa$ controls the sensitivity of the event detection and is driven by the application requirements. Key-frame positions are determined by the local minima in the temporal descriptor at the scale where $K$ equals $\mathrm{K}_{\text {opt }}$. Being located at the local troughs of motion activity, the key-frames will have maximum probability of avoiding motion blur and other artefacts due to object motion or camera work. In addition, the most representative information will be conveyed by the keyframes in areas with no camera work, since the cameraman tends to focus on the main object of interest using a static camera. In case the level of detail required cannot be achieved by using DCE simplification, i.e. initial cost of DCE simplification is too high, algorithm switches to a higher layer of scalable video and generates more detailed metric for a given section of video.

\section{GRAPH BASED FRAME CLUSTERING}

Having the user's experience at the centre of our browsing interface design task, our main aim is to generate an intuitive video summary by conveying the significance of a shot from analysed videos via the size of its key-frame representation. In our case, the objective is to clearly present visual content that is dominant throughout the analysed section of the video, as well as to highlight some unanticipated content.

Following the approach described above, where the size of a key-frame represents its summarisation significance, a cost function $C(i)$ that represent the desired frame size in the final layout is generated, where $C(i) \in[0,1]$ for $i=1, \ldots, N$, and $N$ is the number of extracted key-frames for a given sequence. In order to evaluate the cost function in a way that will support the user's visual experience of the final layout, the clustering based on perceptual similarity is used. More specifically, an efficient graph based clustering method [9] that utilises $18 \times 3 \times 3 \mathrm{HSV}$ colour histogram is applied. 
This approach enables unsupervised analysis of inherent structure of the key-frame data and it copes well with nonlinearity of cluster shapes. The exploited graph-clustering algorithm defines a predicate for measuring the evidence for a boundary between two clusters using a graph-based representation of the image set. Nodes in the constructed graph are the extracted key-frame images, while their histogram difference values are assigned to graph edges. An important characteristic of the method is its ability to preserve detail in low-variability clusters while ignoring detail in highvariability image sets. This algorithm runs in time nearly linear to the number of graph edges, and though we have taken into consideration fully connected image set, due to relatively small number of images the processing is very fast.

The main objective in the process of analysing the inherent structure of the key-frame dataset is to avoid data dependent parameterisation, achieve low algorithm complexity and cope well with high non-linearity of underlying data clusters. In our previous work, we have utilised a specific unsupervised spectral clustering approach [10], but the high complexity of the eigen-decomposition stage of the affinity matrix hindered overall efficiency for large datasets.

To achieve algorithm complexity nearly linear to the number of key-frames, a specific graph based clustering algorithm is utilised [9]. Although initially formulated in the image segmentation context, this algorithm can be extended to a more generic dataset scenario. Its ability to preserve detail in low-variability clusters while ignoring detail in high-variability regions maintains notion of global features of the dataset in the process of making greedy decisions locally.

Following a common approach to graph based image clustering, this method forms edges of a graph $G=(V, E)$, where each image corresponds to a node $v_{i} \in V$ in the graph, and certain images are connected by undirected edges $\left(v_{i}, v_{j}\right) \in E$. Weights on each edge $w\left(v_{i}, v_{j}\right)$ measure the dissimilarity between the two corresponding images.

The graph node grouping is defined by a graph predicate $D\left(c_{1}, c_{2}\right)$, which evaluates if the two regions $c_{1}$ and $c_{2}$ should stay disconnected by comparing inter and intra regional differences:

$$
D\left(c_{1}, c_{2}\right): \operatorname{Ext}\left(c_{1}, c_{2}\right)>\operatorname{mint}\left(c_{1}, c_{2}\right)
$$

The internal difference of a component $c$ is defined as the largest weight in the minimum spanning tree of the component. The joint internal difference measure $\operatorname{mInt}\left(c_{1}, c_{2}\right)$ is given as:

$$
m I n t=\min \left(\operatorname{lnt}\left(c_{1}\right)+\tau\left(c_{1}\right), \operatorname{lnt}\left(c_{2}\right)+\tau\left(c_{2}\right)\right)
$$

The threshold function $\tau(c)=k /|c|$, where $k$ is some constant parameter and $|c|$ denotes the size of $c$, controls the degree to which the difference between the two components must be greater than their internal differences. The intra component difference is defined as the minimal weight edge connecting the two components. The technique adaptively adjusts the merging criterion based on the degree of variability in neighbouring regions of the dataset.

The node grouping is iteratively repeated until there is no more component merging. In order to represent the dominant content of the selected section of video, each component is represented with a frame closest to the component's centre of the mass. Therefore the highest cost function $C(i)=1$ is assigned for $d=0$, where $d$ is the distance of the key-frame closest to the centre of component and $\sigma_{i}$ is $i^{\text {th }}$ frame's component variance. Other members of the component are given values:

$$
C(i)=\alpha \cdot\left(1-e^{-\frac{d i I^{2}}{2 \alpha^{2}}}\right) \cdot h_{\operatorname{ma\alpha }}
$$

The cost function is scaled to have a maximum value $h_{\max }$ in order to be normalised to frame sizes available in the final layout. Parameter $\alpha$ can take values $\alpha \in[0,1]$, and in our case is chosen empirically to be 0.7 . In Figure 1 , a range of different cost dependency curves are depicted for values $\alpha \in\{0.5-1.0\}$ and $h_{\max }=1$. The value of $\alpha$ controls the balance between the importance of the cluster centre and the outliers.

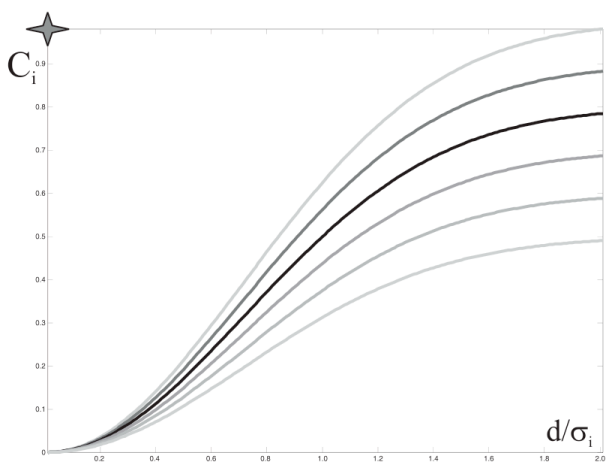

Fig. 1. Importance measure assigned to the frames depending on their distance from the cluster centre

By doing this, cluster outliers (i.e. cutaways, establishing shots, etc.) are presented as more important and attract more attention of the user than key-frames concentrated around the cluster centre. This grouping around the cluster centres is due to common repetitions of similar content in raw video rushes, often adjacent in time. To avoid the repetition of content in the final summary, a set of similar frames is represented by a larger representative, while the others are assigned a lower cost function value.

\section{GENERATING VISUAL SUMMARY}


The main task of the layout module is to generate a visual summary that optimally follows the values of the cost function by using only frame sizes available in comic-like panel templates. The precision of approximation depends upon the maximum height of a panel $h_{\max }$, which gives granularity of the solution. For a given $h_{\max }$, a set of panel templates is generated, assigning a vector of frame sizes to each template.

For a given cost function $C(i)$, there will be a finite number of frame-size values $\Theta(i)$ in the final layout. An example of a single-row layout approximation for $h_{\max }=4$ is depicted in Figure 2, comparing the values of the cost function $C(i)$ with the achieved values of frame sizes $\Theta(i)$. The templates follow the narrative structure of a comic book, while maintaining the original aspect ratio of images forming the panel [10].

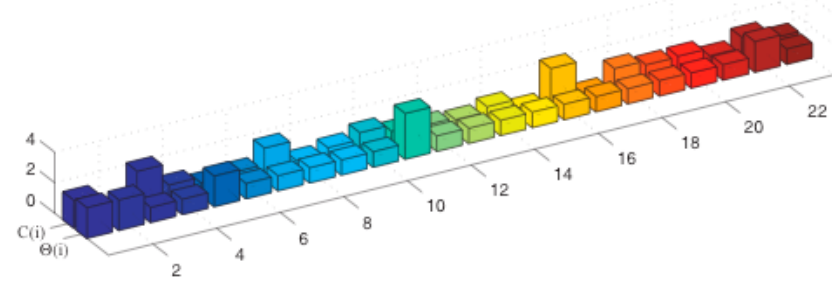

Fig. 2. Approximation of the cost function $C(i)$ by frame sizes $\Theta(i)$ in the final layout for a single row layout.

Since the aim here is to optimally utilise the available space given the required sizes of images, this is a problem of discrete optimisation. However, unlike thoroughly explored discrete optimisation methods like stock cutting or bin packing [11], there is a non-linear transformation layer of panel templates between the error function and available resources. Therefore, a sub-optimal solution using dynamic programming is proposed. It follows a typical structure of the DP algorithm by efficiently finding the solution to an optimisation problem in case the variables in the evaluation function are not interrelated simultaneously. Although there is an indirect dependency between non-adjacent panels due to the fact that the width of the last panel is directly dependent upon the sum of widths of previously used panels, by introducing specific corrections to the DP error function [12] the sub-optimal solution often achieves optimal results. This correction assigns additional cost if the layout needs resizing in order to fit to the required width.

\section{EXPERIMENTAL RESULTS}

The evaluation experiments were conducted using the TRECVID 2006 evaluation content. This content is provided as the benchmarking material for evaluation of video retrieval systems. The videos were transcoded to scalable video format, generating 5 temporal layers and using 4 levels of temporal filtering. Therefore the motion information associated to the lowest temporal level corresponds to every $16^{\text {th }}\left(2^{4}\right)$ frame. Motion activity metrics computed from this lowest layer are used to initialise the DCE stage of the keyframe selection algorithm.

The results depicted in the Figure 3 represent a scale space at four stages of the DCE simplification process, applied to the motion activity metric $\alpha^{0}$ which corresponds to the video bit-stream layer with the lowest available framerate (top of Figure 3). The frame numbers in Figure 3 are related to the original sequence while the number of actual samples of the motion activity metric is 16 times lower.

Gradual removal of less important features of the metric curve is performed using DCE, Figure 3, where $i$ in $a^{i}$ denotes the DCE simplification stage. The bottom curve $a^{3}$ from Figure 3 is used to select the key-frames at the locations of curve troughs. The key-frames are then used for modelling of a summary.
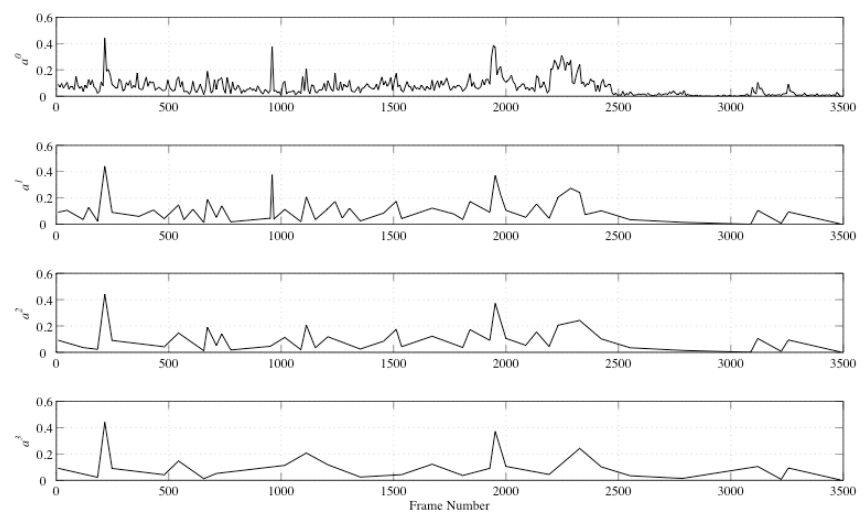

Fig. 3. Scale space of the temporal descriptor generated by the DCE algorithm for the sequence summaries in Figure 2.

An example of the final layout obtained from the layered bit-streams using proposed layout algorithm is presented in Figure 5. The results are obtained from the same set of key-frames targeting different heights of panels. One can observe that the spatial compression of the layouts depends on selected height $h_{\max }$, since the larger $h_{\max }$ values tend to allow more compact representations.

Without loosing the notion of temporal structure as well as representing every detail of the content, the resulting video summary achieves spatial compression ration of $2 / 5$ while producing visually pleasant experience for the user. The layout algorithm complexity is evaluated by comparing the processing speed with the methods that utilise comiclike narrative structure in video summarisation, which are presented in [2] and [3]. Firstly, both methods have been proved unfeasible for summaries with more than 100 frames. The layout processing times for of the algorithms presented in [2] $\mathrm{T}_{\mathrm{OR}}$ and in [3] $\mathrm{T}_{\mathrm{FS}}$, compared to the proposed method $\mathrm{T}_{\mathrm{DP}}$ are numerically given in Table 1. From the results shown it can be observed that the utilised method achieves linear complexity, as depicted in Figure 4. Therefore, this method proves to be suitable for fast summarisation targeting responsive browsing interfaces. 


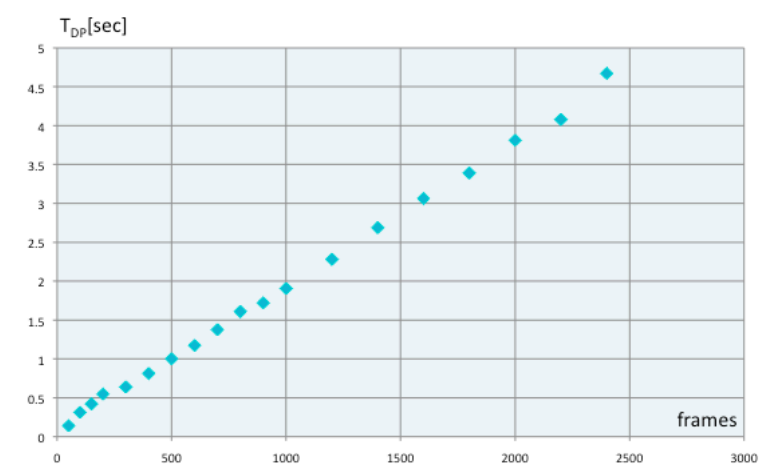

Fig. 4. Dependency of the layout duration $T_{D P}$ on the number of frames in the summary

Table 1. Layout duration in seconds for a given number of frames $N$.

\begin{tabular}{|c||c|c|c|c|c|c|}
\hline$N$ & 25 & 75 & 125 & 150 & 1000 & 2500 \\
\hline \hline $\mathrm{T}_{\mathrm{OR}}$ & 0.03 & 0.16 & 1.8 & $\mathrm{X}$ & $\mathrm{X}$ & $\mathrm{X}$ \\
\hline $\mathrm{T}_{\mathrm{FS}}$ & 0.03 & 0.57 & 200 & $\mathrm{X}$ & $\mathrm{X}$ & $\mathrm{X}$ \\
\hline $\mathrm{T}_{\mathrm{DP}}$ & 0.04 & 0.13 & 0.32 & 0.44 & 1.07 & 4.20 \\
\hline
\end{tabular}

\section{CONCLUSIONS}

The experimental results show that the utilisation of the layered bit-stream and compressed domain motion features, coupled with a fast and robust curve simplification, the system efficiently extracts the most representative set of keyframes. Exploiting the narrative structure of comics and using its well-known intuitive rules, visual summaries are generated in a user centred way. Not only does this approach improves the processing time of the summarisation task, but it enables new functionalities of visualisation for large-scale video archives, such as real-time interaction and relevance feedback.

\section{REFERENCES}

[1] R. Dony, J. Mateer, J. Robinson, "Techniques for automated reverse storyboarding," IEE Proc. Vision, Image and Signal Processing, Vol. 152, No. 4, pp. 425 - 436, 2005.

[2] S. Uchihashi, J. Foote, A. Girgensohn, J. Boreczky, "Video manga: generating semantically meaningful video summaries," Proc. 7th ACM Int'l Conference on Multimedia, pp. 383 $392,1999$.

[3] A. Girgensohn, "A fast layout algorithm for visual video summaries," Proc Int'l Conference on Multimedia and Expo, Vol. 2, pp. 77 - 80, 2003.

[4] Video Mining, A Rosenfeld, D. Doermann, D. DeMenthon (Editors), Kluwer Academic Publishers, 2003.

[5] N. Adami, A. Signoroni, R. Leonardi, "State-of-the-art and trends in scalable video compression with wavelet-based approaches," IEEE Trans. on Circ. and Sys. for Video Tech., Vol. 17, Iss. 9, pp. 1238 - 1255, Sept. 2007.

[6] M. Mrak, N. Sprljan, E. Izquierdo, "Motion estimation in temporal subbands for quality scalable motion coding," Electronics Letters, No. 41, pp. 1050 - 1051, 2005.

[7] H. Schwarz, D. Marpe, T. Wiegand, "Overview of the scalable video coding extension of the H.264/AVC standard," IEEE Trans. on Circ. and Sys. for Video Tech., Vol. 17, Iss. 9, pp. 1103 - 1120, Sept. 2007.

[8] L. J. Latecki, R. Lakamper, "Convexity rule for shape decomposition based on discrete contour evolution," Computer Vision and Image Understanding, Vol. 73, pp. 441-454, 1999.

[9] P. F. Felzenszwalb and D. P. Huttenlocher, "Efficient GraphBased Image Segmentation", International Journal of Computer Vision, Volume 59, Number 2, September 2004.

[10] J. Calic and N. W. Campbell, "Compact Visualisation of Video Summaries," EURASIP Journal on Advances in Signal Processing, vol. 2007, Article ID 19496, 2007.

[11] A. Lodi, S. Martello, M. Monaci, "Two-dimensional packing problems: A survey," European Journal of Operational Research, Vol. 141, Iss. 2, pp. 241 - 252, 2002.

[12] J. Calic, D. P. Gibson, and N. W. Campbell, "Efficient layout of comic-like video summaries," IEEE Trans. on Circ. and Sys. for Video Tech., Vol. 17, Iss. 7, pp. 931 - 936, July 2007.

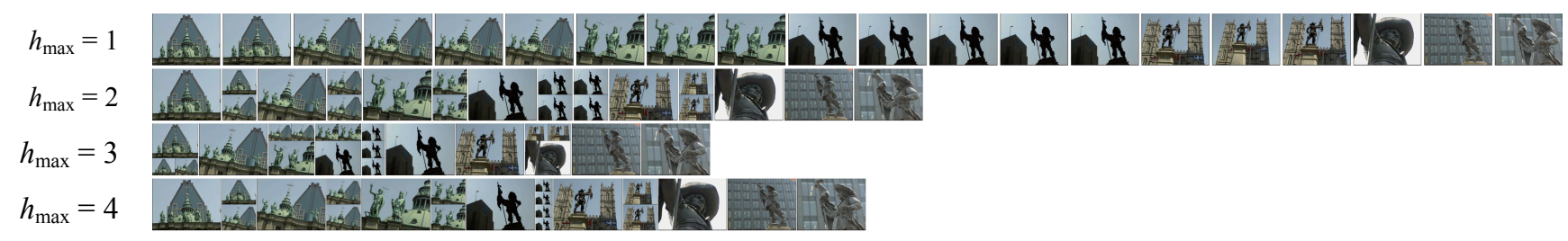

a) Resulting layouts using panels with different heights

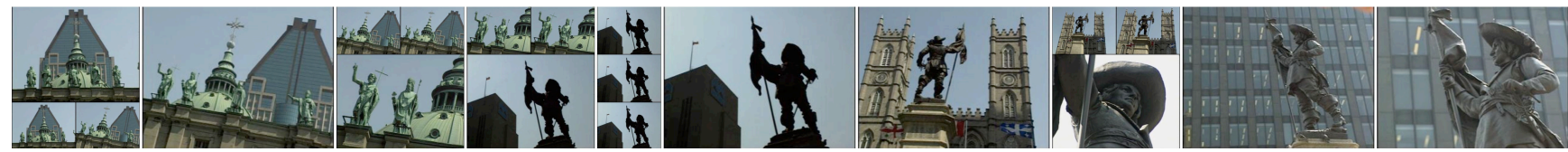

b) Enlarged layout from $h_{\max }=3$

Fig. 5. Resulting video summaries for different panel heights. 